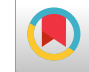

\title{
Prevalence and Severity of Thrombocytopenia in Blood Culture Proven Neonatal Sepsis: A Prospective Study
} Ramesh Bhat Y., ${ }^{1,}$ Phalguna Kousika, ${ }^{1}$ Leslie Lewis, ${ }^{1}$ and Jayashree Purkayastha ${ }^{1}$

${ }^{1}$ Department of Pediatrics, Kasturba Medical College, Manipal University, Manipal-576104, Karnataka, India

"Corresponding author: Ramesh Bhat Y., Department of Pediatrics, Kasturba Medical College, Manipal University, Manipal-576104, Karnataka, India. E-mail: docrameshbhat@yahoo.co.in

Received 2017 March 18; Revised 2017 July 03; Accepted 2017 August 19.

\begin{abstract}
Background: The reported prevalence and pattern of thrombocytopenia in neonatal sepsis vary widely.

Objectives: We aimed to determine the prevalence and severity of thrombocytopenia in blood culture proven neonatal sepsis.

Methods: The study was conducted in a University hospital by recruiting neonates with sepsis in whom blood culture had grown microorganisms. The initial platelet count refers to the one obtained at the same time as the positive blood culture. Platelet counts were monitored 12 - 24 hourly. Thrombocytopenia was considered mild if between $50,000 / \mathrm{mm}^{3}$ and $150,000 / \mathrm{mm}^{3}$, moderate if between $20,000 / \mathrm{mm}^{3}$ and $50,000 / \mathrm{mm}^{3}$, and severe if $<20,000 / \mathrm{mm}^{3}$ or $<50,000 / \mathrm{mm}^{3}$ with clinical bleeding.

Results: A total of 143 episodes of blood culture proven sepsis in 131 neonates were studied. Gram positive bacteria identified in $33.6 \%$, gram negative bacteria in $53.8 \%$, and fungi in $12.6 \%$. Klebsiella predominated among Gram negative bacteria (39\%) and Candida species (94.4\%) among fungi. Initial thrombocytopenia was observed in 84 (58.7\%) episodes; it was mild, moderate, and severe in $39.3 \%$, 25\%, and $35.7 \%$ respectively. Initial thrombocytopenia among Gram positive, Gram negative, and fungal sepsis were $41.7 \%$, $70.1 \%$, and $55.6 \%$, respectively. Severe thrombocytopenia in the respective groups was $20 \%, 44.4 \%$ and $20 \%$. Overall ( $80 \%$ ) and severe thrombocytopenia (45.8\%) was highest in Klebsiella sepsis. Thrombocytopenia was moderate in $60 \%$ of Candida sepsis. An additional 23.7\% had thrombocytopenia subsequently. In $51.2 \%$, thrombocytopenia persisted beyond 3 days.

Conclusions: Thrombocytopenia was observed in $58.7 \%$ of culture proven neonatal sepsis. Initial thrombocytopenia was common among Gram negative sepsis and mostly of a moderate degree in Candida sepsis.
\end{abstract}

Keywords: Neonates, Sepsis, Thrombocytopenia, Blood Culture, Bacteria

\section{Background}

Thrombocytopenia in neonates, defined mostly as platelet count less than $150,000 / \mathrm{mm}^{3}$ regardless of gestational age, is said to affect $1 \%-5 \%$ of neonates at birth and up to 50\% among those receiving intensive care (1). Thrombocytopenia is considered as a risk factor for haemorrhage, especially intraventricular in location, mortality, and adverse neurodevelopmental outcome (1). Sepsis remains an important cause of neonatal thrombocytopenia (2-7). However, the reported prevalence and severity of thrombocytopenia in neonatal sepsis varies widely. Higher rates of thrombocytopenia were reported in late-onset sepsis (59.3\%) compared to early-onset sepsis (24\%)(2) as well as in the preterm and low-birth weight neonates. Thrombocytopenia of 54\% in culture-proven sepsis episodes was reported among very low birth weight (VLBW) neonates (4). Furthermore, Gram negative and fungal infections compared to gram positive infections had significantly higher incidence of thrombocytopenia (2-4). In another study (5), rates of thrombocytopenia in coagulase negative Staphylococcus sepsis, Klebsiella sepsis and isolated fungal sepsis were $33.3 \%, 60 \%$, and $66 \%$, respectively. A spanish study reported thrombocytopenia in 100\% of Candida sepsis (7). An Indian study (5) reported thrombocytopenia in 59.5\% of nosocomial sepsis with mild, moderate, and severe thrombocytopenia in $27 \%, 20 \%$, and $12.5 \%$, respectively.

The duration of sepsis related thrombocytopenia varies with causative organisms. Gram negative and fungal infections had a longer duration of thrombocytopenia compared to Gram positive infections (4). In addition, the mortality in fungal sepsis was higher when it was associated with thrombocytopenia than without (4-7). Thrombocytopenia related to late-onset sepsis was found to be severe and associated with higher morbidity and mortality (5). 


\section{Objectives}

In the above context, we aimed to study the pattern and severity of thrombocytopenia by enrolling neonates with blood culture proven sepsis.

\section{Methods}

\subsection{Study Subjects}

We enrolled neonates at Kasturba Medical college hospital with clinically diagnosed sepsis, prospectively from March 2014 to July 2015. Neonates were included in the study if their blood culture grew microorganisms. The study protocol was approved by the institutional ethics committee. Neonates with other known causes of thrombocytopenia such as maternal thrombocytopenia, HELLP syndrome, SLE, preeclampsia and eclampsia, congenital infections, polycythemia, and necrotising enterocolitis were excluded. Neonates with IUGR, especially those born to PIH mothers and TORCH infections who are likely to have thrombocytopenia, have also been excluded. Neonates in whom blood culture grew contaminants, or 2 or more microorganisms were also excluded. Neonates who stayed for less than 3 days in whom the 2 nd platelet count could not be obtained were excluded from analysis.

\subsection{Blood Culture and Isolation of Microorganisms}

Blood sample was processed using BacT alert automated system containing brain heart infusion biphasic medium. Subcultures were done on Sheep blood agar and MacConkey agar at the earliest visual detection of turbidity or blindly on days 1, 4, and 7 if the bottles did not show turbidity. Gram's staining was performed to identify the microorganisms. Antimicrobial susceptibility was performed using the Kirbey Bauer disc diffusion method according to the Clinical and Laboratory Standard Institute guidelines and with an automated Vitek 2 system. The microorganisms were divided into 3 groups: Gram positive, Gram negative, and fungal.

\subsection{Platelet Counts}

Platelet counts were obtained on a coulter counter (Beckman Coulter-COULTER@ LH 750 Hematology Analyzer). All platelet counts less than $150,000 / \mathrm{mm}^{3}$ were rechecked manually. We have taken the first platelet count that refers to the platelet count on blood sample taken at the same time as the sample for blood culture. We meant this as the initial platelet count and related the trend of platelet counts to this point. Subsequently, platelet counts were monitored 12 - 24 hours. The 2 nd platelet count refers to the one obtained at $72 \pm 12$ hours after the initial platelet count. Severity of thrombocytopenia was classified as mild when the platelet count was between $50,000 / \mathrm{mm}^{3}$ and $150,000 / \mathrm{mm}^{3}$, moderate when platelet count was between $20,000 / \mathrm{mm}^{3}$ and $50,000 / \mathrm{mm}^{3}$, and severe when platelet count was $<20,000 / \mathrm{mm}^{3}$ or $<50000 / \mathrm{mm}^{3}$ with clinical bleeding. Platelet transfusions were given if platelet count was below $20,000 / \mathrm{mm}^{3}$ or if there was clinical bleeding. Effect of platelet transfusion on platelet count was not studied. We have fairly excluded the known causes of neonatal thrombocytopenia while studying sepsis and hence normal controls were not taken.

\subsection{Statistical Analysis}

Data was analyzed using statistical the software package, SPSS version 16. Platelet counts were analyzed among the organism groups as well as among individual organism. Association of severity of thrombocytopenia among different groups was assessed using Chi Square test.

\section{Results}

Of 1024 episodes of suspected sepsis, 215 episodes (21\%) grew microorganism in blood culture. After excluding 72 episodes (Figure 1), 143 sepsis episodes in 131 neonates were further analyzed.

The baseline characteristics of 131 neonates are shown in Table 1. About 60 (45.8\%) were term neonates and 70 (53.4\%) were preterm. About 41 had low birth weight, 25 had VLBW, and 20 had ELBW. Male neonates constituted 64.1\% and outborn neonates, $38.9 \%$.

About 111 (77.6\%) were late-onset and 32 (22.4\%) were early- onset sepsis. Of the late-onset sepsis, 65 (58.6\%) episodes were due to Gram negative bacteria, 31 (27.9\%) were due to Gram positive bacteria, and 15 (13.5\%) were fungal infections. In 32 episodes of early-onset sepsis, 17 (53.1\%) were gram positive, 12 (37.5\%) were Gram negative and 3 (9.4\%) were fungal sepsis.

Overall Gram negative, Gram positive, and fungal sepsis were $53.8 \%, 33.6 \%$, and $12.6 \%$, respectively (Table 2 ). Among Gram negative organisms, Klebsiellapneumoniae was the commonest organism isolated (39\%). Candida species constituted $94.4 \%$ of fungal infections.

Of the total 143 sepsis episodes, 58.7\% had thrombocytopenia at the time of blood sample drawn for culture (Table 3). This (initial) thrombocytopenia was severe in $35.7 \%$. The median platelet count among thrombocytopenic episodes was $37,000 / \mathrm{mm}^{3}$ [IQR :(19250, 71500)]. The lowest value was $6,000 / \mathrm{mm}^{3}$.

Higher percentages of Gram negative sepsis (70.1\%) had initial thrombocytopenia compared to gram positive sepsis $(41.7 \%)$ and fungal sepsis $(55.6 \%)(P=0.01)$. Severe initial 


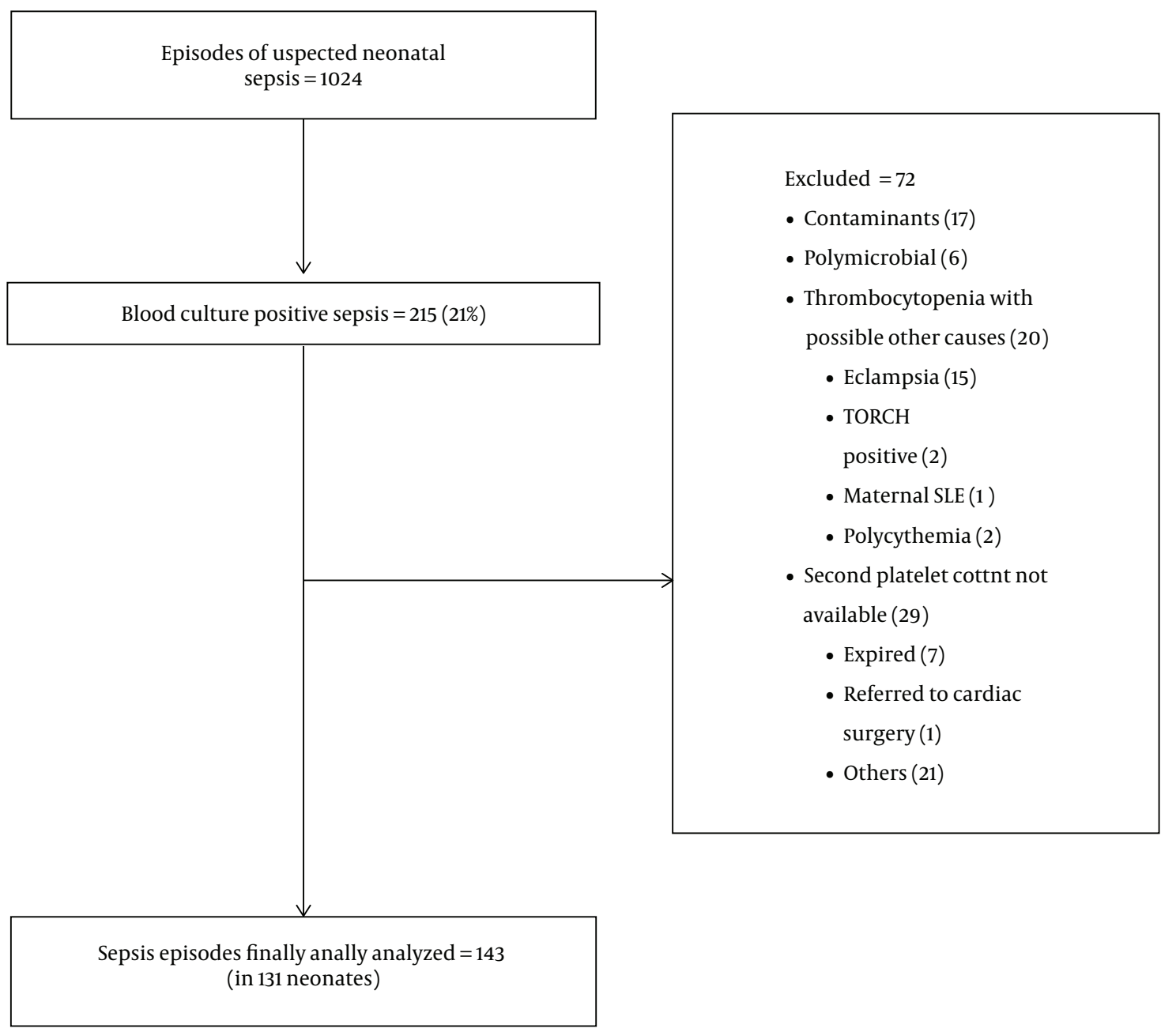

Figure 1. Study Flow Chart

thrombocytopenia was observed in 24 episodes (44.4\%) of Gram negative sepsis. Initial thrombocytopenia was moderate in $60 \%$ of fungal sepsis (Table 4 ).

About $63.3 \%$ of term and $51.4 \%$ of preterm neonates had initial thrombocytopenia. Significantly higher percentage of preterm infants had severe thrombocytopenia compared to term infants $(47.2 \%$ vs $21.1 \%)(P=0.01)$. Initial thrombocytopenia was highest (65\%) among ELBW neonates.

Of $K$. pneumoniae sepsis, $80 \%$ had initial thrombocytopenia, $45.8 \%$ of them were severe thrombocytopenia. Thrombocytopenia was observed in $80 \%$ of Burkholderia cepaciae, $66.7 \%$ of Acinetobacter sepsis, $64.7 \%$ of Enterobacter, $47.1 \%$ of CoNS sepsis, and $58.8 \%$ of Candida sepsis (Table 5).

Among neonates with initial thrombocytopenia, 84.6\% episodes continued to have thrombocytopenia after 3 days, $51.2 \%$ with same severity, and $21.4 \%$ with worsened count. About 23.7\% (14/59) of culture positive neonatal sepsis with normal initial platelet count had thrombocytopenia subsequently, 35.1\% among them had severe thrombocytopenia.

Thrombocytopenia persisted beyond 3 days in 16 out of 20 (80\%) episodes of Gram positive, 47 out of 54 (87\%) 
Table 1. Baseline Characteristics of Study Neonates $(n=131)$

\begin{tabular}{|cc}
\hline Characteristics & No. (\%) \\
\hline Gestational age & \\
\hline Term & $60(45.8)$ \\
\hline Preterm & $70(53.4)$ \\
\hline Post term & $1(0.8)$ \\
\hline Birth weight & \\
\hline Normal & $45(34.4)$ \\
\hline Low birth weight & $41(31.3)$ \\
\hline Very low birth weight & $25(19.1)$ \\
\hline Extremely Low birth weight & $20(15.3)$ \\
\hline Gender & $84(64.1)$ \\
\hline Male & $47(35.9)$ \\
\hline Female & $80(61.1)$ \\
\hline Place of Delivery & $51(38.9)$ \\
\hline Inborn & \\
\hline Outborn & \\
\hline
\end{tabular}

Table 2. Distribution of Blood Culture Isolates $(n=143)$

\begin{tabular}{|c|c|}
\hline & Number of Episodes of Sepsis (\%) \\
\hline \multicolumn{2}{|l|}{ Gram Positive bacteria $(n=48)$} \\
\hline CoNS & $34(70.8)$ \\
\hline MRSA & $4(8.3)$ \\
\hline S. aureus & $4(8.3)$ \\
\hline Streptococcus & $4(8.3)$ \\
\hline Enterococcus & $2(4.2)$ \\
\hline \multicolumn{2}{|l|}{ Gram Negative bacteria $(\mathbf{n}=77)$} \\
\hline Klebsiella pneumoniae & $30(39)$ \\
\hline Enterobacter spp. & $17(22.1)$ \\
\hline Pseudomonas aeruginosa & $6(7.8)$ \\
\hline E. coli & $6(7.8)$ \\
\hline Acinetobacter spp. & $6(7.8)$ \\
\hline Burkholderia cepaciae & $5(6.5)$ \\
\hline NFGNB & $5(6.5)$ \\
\hline Serratia marcescens & $2(2.6)$ \\
\hline \multicolumn{2}{|l|}{ Fungal $(n=18)$} \\
\hline Candida & $17(94.4)$ \\
\hline Kodamaea spp. & $1(5.6)$ \\
\hline
\end{tabular}

Abbreviations: CoNS, coagulase negative Staphylococcus; MRSA, methicillin resistant Staphylococcus aureus; NFGNB, non fermenting gram negative Bacilli.
Table 3. Platelet Count at the Time of Drawing Blood Culture $(n=143)$

\begin{tabular}{l|c|c}
\hline \multirow{2}{*}{ Platelet count } & Normal & $\begin{array}{c}\text { Number of Episodes } \\
\text { of Sepsis (\%) }\end{array}$ \\
\cline { 2 - 3 } & $\begin{array}{c}\text { Low } \\
\text { (thrombocytopenia) }\end{array}$ & $59(41.3)$ \\
\hline \multirow{2}{*}{$\begin{array}{l}\text { Severity of } \\
\text { thrombocytopenia }\end{array}$} & Mild & $84(58.7)$ \\
\cline { 2 - 3 } & Moderate & $33(39.3)$ \\
\cline { 2 - 3 } & Severe & $21(25.0)$ \\
\hline
\end{tabular}

episodes of Gram negative, and 8 out of $10(80 \%)$ episodes of fungal sepsis. Three episodes of $K$. pneumoniae sepsis had normal initial platelet count but had severe thrombocytopenia subsequently.

\section{Discussion}

Thrombocytopenia in neonates bothers the treating clinician and if severe presents as management problem (1-3, 8-13). Sepsis remains an important etiology among many causes $(1-5,13)$. Hemorrhage and mortality were reported with severe thrombocytopenia $(1,14)$. Bacterial sepsis associated with thrombocytopenia had increase mortality $(3,14)$. Thrombocytopenia has been reported as an independent risk factor for sepsis related death among VLBW infants (15). Increased platelets destruction, an impaired platelet production or a combination may be the underlying mechanism of thrombocytopenia $(6,12,16)$. Characterization of sepsis related thrombocytopenia in neonates is much desired as the incidence varies with several factors. The present study explored the prevalence and severity of thrombocytopenia in culture proven neonatal sepsis in a set up from a developing country.

The blood culture yield of $21 \%$ in the present study is higher than reports (16\%) of Guida et al. (4) but lower than reports (37\%) of Bhat et al. (3) and Muley et al. (26.6\%) (17). The predominance of Gram negative sepsis, as identified in this study, is well recognized (18). In a study by Bhat et al. (3), Gram-negative sepsis was observed in 71\%, Gram positive sepsis in $20 \%$, and fungal sepsis in $8.6 \%$. The predominance of Klebsiella spp. isolates agrees with earlier reports from India $(19,20)$. The rate of fungal isolates $(12.6 \%)$ in the present study is similar to a previous report (3). Another study reported higher (19\%) fungal isolates (7).

Among sepsis episodes, 58.7\% had thrombocytopenia initially (at the time of blood sample drawn for culture). Thrombocytopenia was severe in $35.7 \%$. Thrombocytopenia was observed in $67 \%$ of sepsis episodes by Bhat et al. (3) and in $71 \%$ by Guida et al. (4). These observations sug- 
Table 4. Organism Group Specific Initial Platelet Response $(n=143)^{a}$

\begin{tabular}{|c|c|c|c|c|}
\hline & \multirow[t]{2}{*}{ Thrombocytopenia } & \multicolumn{3}{|c|}{ Severity of Thrombocytopenia } \\
\hline & & Mild & Moderate & Severe \\
\hline Gram positive $(n=48)$ & $20(41.7)$ & $12(60.0)$ & $4(20.0)$ & $4(20.0)$ \\
\hline Gram negative $(n=77)$ & $54(70.1)$ & $19(35.2)$ & $11(20.4)$ & $24(44.4)$ \\
\hline Fungal ( $n=18$ ) & $10(55.6)$ & $2(20.0)$ & $6(60.0)$ & $2(20.0)$ \\
\hline
\end{tabular}

${ }^{\mathrm{a}}$ Values are expressed as No. (\%).

Table 5. Initial Platelet Response to Common Microorganisms ${ }^{\mathrm{a}}$

\begin{tabular}{|c|c|c|c|c|}
\hline & \multirow[t]{2}{*}{ Thrombocytopenia } & \multicolumn{3}{|c|}{ Severity of Thrombocytopenia } \\
\hline & & Mild & Moderate & Severe \\
\hline K. pneumoniae $(\mathbf{n}=30)$ & $24(80.0)$ & $6(25.0)$ & $7(29.1)$ & $11(45.8)$ \\
\hline Burkholderia cepaciae $(n=5)$ & $4(80.0)$ & $2(50.0)$ & 0 & $2(50.0)$ \\
\hline $\operatorname{NFGNB}(\mathbf{n}=5)$ & $4(80.0)$ & $2(50.0)$ & $1(25.0)$ & $1(25.0)$ \\
\hline $\operatorname{MRSA}(n=4)$ & $3(75.0)$ & $1(33.3)$ & $1(33.3)$ & $1(33.3)$ \\
\hline Acinetobacter spp. $(\mathrm{n}=6)$ & $4(66.7)$ & 0 & $2(50.0)$ & $2(50.0)$ \\
\hline E. $\operatorname{coli}(\mathbf{n}=6)$ & $4(66.7)$ & $2(50.0)$ & 0 & $2(50.0)$ \\
\hline Enterobacter spp. $(\mathbf{n}=17)$ & $11(64.7)$ & $6(54.5)$ & $1(9.0)$ & $4(36.3)$ \\
\hline Candida $(\mathbf{n}=17)$ & $10(58.8)$ & $2(20.0)$ & $6(60.0)$ & $2(20.0)$ \\
\hline P. aeruginosa $(\mathrm{n}=6)$ & $3(50.0)$ & $1(33.3)$ & 0 & $2(66.7)$ \\
\hline $\operatorname{CoNS}(n=34)$ & $16(47.1)$ & $10(62.5)$ & $3(18.7)$ & $3(18.7)$ \\
\hline Staphylococcus aureus $(\mathrm{n}=4)$ & $1(25.0)$ & $1(100)$ & 0 & 0 \\
\hline
\end{tabular}

Abbreviations: CoNS, coagulase negative Staphylococcus; MRSA, methicillin resistant Staphylococcus aureus; NFGNB, non fermenting gram negative Bacilli.

${ }^{\mathrm{a}}$ Values are expressed as No. (\%).

gest that thrombocytopenia is frequent in neonatal sepsis. Neonates respond to sepsis by up-regulating Tpo production and thrombopoiesis, however, the degree of upregulation is said to be only modest. Thrombocytopenia is likely to occur when the rate of platelet consumption exceeds the rate of platelet production (16).

Significantly higher percentage of preterm infants had severe thrombocytopenia compared to term infants ( $47.2 \%$ vs $21.1 \%)$. The frequency of initial thrombocytopenia was highest among ELBW neonates. Previously, Charoo et al. (5) studied 200 VLBW neonates with sepsis and reported thrombocytopenia in 59.5\%. A limited response to thrombocytopenia, in terms of platelet production and thrombopoietin in VLBW neonates, especially during sepsis with a decreased energy reserves in the host, may be responsible for increased frequency of thrombocytopenia (21).

The degree of thrombocytopenia varies with causative microorganisms. In the present study, significantly higher percentages of gram negative sepsis (70.1\%) had initial thrombocytopenia compared to gram positive sepsis
(41.7\%) and fungal sepsis (55.6\%). Severe initial thrombocytopenia was observed in $44.4 \%$ of episodes of Gram negative sepsis. Initial thrombocytopenia was moderate in $60 \%$ of fungal sepsis. This observation agrees with the previous report that thrombocytopenia was more frequent in gram negative sepsis (4). Increased platelet destruction through antibody mediated binding, uptake, and activation are proposed as the likely mechanisms of thrombocytopenia associated with Gram-negative sepsis. Cell-free extracts containing lipopolysaccharide and a component of the cell wall of Gram negative organisms have been shown to directly induce thrombocytopenia in animal models (22).

In the present study, K. pneumoniae sepsis had the highest initial thrombocytopenia (80\%), $45.8 \%$ of them being severe thrombocytopenia. The frequency of thrombocytopenia was $80 \%$ in Burkholderia cepaciae, 66.7\% in Acinetobacter spp., $64.7 \%$ in Enterobacter spp., and 58.8\% in Candida spp. sepsis. The frequency was lower (47.1\%) in CoNS sepsis. Organism specific platelet response in neonatal sepsis is char- 
acterized to some extent by previous researchers. Charoo et al. (5) found $60 \%$ of K. pneumoniae sepsis having thrombocytopenia. Thrombocytopenia in Enterobacter spp. sepsis varied from $65.5 \%$ to $85.7 \%$ in previous studies $(6,23)$.

The prevalence of thrombocytopenia in CoNS sepsis in our study is higher than that (33\%) reported by Charoo et al. (5). In contrast, Kashu et al. found thrombocytopenia in $84 \%$ of neonates with persistent CoNS bacteremia (24). Thrombocytopenia is frequent in fungal sepsis. Charoo et al. reported that $66 \%$ of fungal sepsis had thrombocytopenia (5). Torres et al. found thrombocytopenia in $100 \%$ of Candida sepsis (7) and concluded that thrombocytopenia is a highly specific marker of fungal sepsis. We observed lower rates of thrombocytopenia in sepsis due to Candida spp.

When we studied the course of thrombocytopenia about $23.7 \%$ of neonatal sepsis with normal initial platelet counts were found to have thrombocytopenia subsequently, of which $35.1 \%$ were severe in nature. Overall, a large majority (84.6\%) of neonatal sepsis with initial thrombocytopenia continued to have thrombocytopenia until 3 days with $51.2 \%$ at the same severity and $21.4 \%$ with worsened platelet counts. Thrombocytopenia persisted even after 3 days in $80 \%$ of episodes due to Gram positive, $87 \%$ of episodes due to Gram negative, and $80 \%$ of episodes due to fungal sepsis. Three episodes of K. pneumoniae sepsis, with normal initial platelet counts, had severe thrombocytopenia subsequently. These observations suggest the need for continuous monitoring of platelet counts in neonates with sepsis. Previous studies reported the mean duration of thrombocytopenia in Gram negative and fungal infections as 2-8 days and in Gram positive infections as $0.4-2.8$ days (4).

Our study had few limitations. Although, frequent monitoring of platelet counts (12th hourly) could yield better information on course of thrombocytopenia, we did not adopt this policy in all neonates due to financial constraints. Similarly, we could not obtain the platelet counts beyond day 3 in all neonates, although we continued monitoring platelet counts when it was low.

\section{Conclusions}

Due to the fact that sepsis remains an important cause of neonatal thrombocytopenia and the degree of thrombocytopenia in sepsis varies from mild to severe, it warrants monitoring. More than half of the blood culture proven sepsis episodes having thrombocytopenia at an early stage informs the magnitude of the problem. Higher percentages of initial thrombocytopenia among Gram negative sepsis compared to fungal and Gram positive sepsis further characterizes this thrombocytopenia. Sepsis with
Klebsiella, Burkholderia, and Acinetobacter species needs special attention regarding thrombocytopenia. The observations that persistence or worsening of thrombocytopenia beyond 3 days among thrombocytopenic sepsis episodes and new-onset thrombocytopenia in the subsequent period stresses the need for continued monitoring of platelet counts.

\section{Acknowledgments}

Authors thank the head and faculty members in the Department of Pediatrics for their kind help, support, and encouragement throughout the study.

\section{Footnote}

Authors' Contribution: Ramesh Bhat Y. formulated the study, supervised the data collection, interpreted the results, wrote the manuscript, and approved it. Phalguna Kousika was involved in systematic data collection, analysis of results, and manuscript writing. Leslie Lewis and Jayashree Purkayastha gave expert inputs to the study.

\section{References}

1. Roberts I, Murray NA. Neonatal thrombocytopenia: causes and management. Arch Dis Child Fetal Neonatal Ed. 2003;88(5):F359-64. [PubMed: 12937037].

2. Khassawneh M, Khader Y, Abuqtaish N. Clinical features of neonatal sepsis caused by resistant Gram-negative bacteria. Pediatr Int. 2009;51(3):332-6. doi: 10.1111/j.1442-200X.2008.02767.x. [PubMed: 19400829]

3. Bhat MA, Bhat JI, Kawoosa MS, Ahmad SM, Ali SW. Organism-specific platelet response and factors affecting survival in thrombocytopenic very low birth weight babies with sepsis. J Perinatol. 2009;29(10):7028. doi: 10.1038/jp.2009.72. [PubMed: 19554015].

4. Guida JD, Kunig AM, Leef KH, McKenzie SE, Paul DA. Platelet count and sepsis in very low birth weight neonates: is there an organismspecific response?. Pediatrics. 2003;111(6 Pt 1):1411-5. [PubMed: 12777561].

5. Charoo BA, Iqbal JI, Iqbal Q, Mushtaq S, Bhat AW, Nawaz I. Nosocomial sepsis-induced late onset thrombocytopenia in a neonatal tertiary care unit: a prospective study. Hematol Oncol Stem Cell Ther. 2009;2(2):349-53. [PubMed: 20118059].

6. Torkaman M, Afsharpaiman SH, Hoseini MJ, Moradi M, Mazraati A, Amirsalari S, et al. Platelet count and neonatal sepsis: a high prevalence of Enterobacter spp. Singapore Med J. 2009;50(5):482-5. [PubMed: 19495516].

7. Torres Claveras S, Dupla Arenaz M, Perez Delgado R, Aliaga Mazas $\mathrm{Y}$, Rebage Moises V. [Nosocomial Candida infections and thrombocytopenia in very low birth weight newborns]. An Pediatr (Barc). 2007;67(6):544-7. [PubMed: 18053518].

8. Bonifacio L, Petrova A, Nanjundaswamy S, Mehta R. Thrombocytopenia related neonatal outcome in preterms. Indian J Pediatr. 2007;74(3):269-74. [PubMed: 17401266].

9. Roberts I, Stanworth S, Murray NA. Thrombocytopenia in the neonate. Blood Rev. 2008;22(4):173-86. doi: 10.1016/j.blre.2008.03.004. [PubMed: 18433954]. 
10. Murray NA, Howarth LJ, McCloy MP, Letsky EA, Roberts IA. Platelet transfusion in the management of severe thrombocytopenia in neonatal intensive care unit patients. Transfus Med. 2002;12(1):35-41. [PubMed: 11967135].

11. Arif SH, Ahmad I, Ali SM, Khan HM. Thrombocytopenia and bacterial sepsis in neonates. Indian J Hematol Blood Transfus. 2012;28(3):147-51. doi: 10.1007/s12288-011-0118-7. [PubMed: 23997450].

12. Roberts IA, Murray NA. Neonatal thrombocytopenia: new insights into pathogenesis and implications for clinical management. Curr Opin Pediatr. 2001;13(1):16-21. [PubMed: 11176238].

13. Colarizi P, Fiorucci P, Caradonna A, Ficuccilli F, Mancuso M, Papoff P. Circulating thrombopoietin levels in neonates with infection. Acta Paediatr. 1999;88(3):332-7. [PubMed: 10229048].

14. Ahmad MS, Waheed A. Platelet counts, MPV and PDW in culture proven and probable neonatal sepsis and association of platelet counts with mortality rate.J Coll Physicians Surg Pak. 2014;24(5):340-4. [PubMed: 24848393].

15. Levit O, Bhandari V, Li FY, Shabanova V, Gallagher PG, Bizzarro MJ. Clinical and laboratory factors that predict death in very low birth weight infants presenting with late-onset sepsis. Pediatr Infect Dis J. 2014;33(2):143-6. doi: 10.1097/INF.0000000000000024. [PubMed: 24418836].

16. Brown RE, Rimsza LM, Pastos K, Young L, Saxonhouse MA, Bailey $\mathrm{M}$, et al. Effects of sepsis on neonatal thrombopoiesis. Pediatr Res. 2008;64(4):399-404. doi: 10.1203/PDR.0b013e318181ad49. [PubMed: 18552713].

17. Muley VA, Ghadage DP, Bhore AV. Bacteriological Profile of Neonatal Septicemia in a Tertiary Care Hospital from Western India. J Glob Infect Dis. 2015;7(2):75-7. doi:10.4103/0974-777X.154444. [PubMed:
26069427].

18. Zaidi AK, Huskins WC, Thaver D, Bhutta ZA, Abbas Z, Goldmann DA. Hospital-acquired neonatal infections in developing countries. Lancet. 2005;365(9465):1175-88. doi: 10.1016/S0140-6736(05)71881-X. [PubMed: 15794973].

19. Bhat YR, Lewis LE, KEV. Bacterial isolates of early-onset neonatal sepsis and their antibiotic susceptibility pattern between 1998 and 2004: an audit from a center in India. Ital J Pediatr. 2011;37:32. doi: 10.1186/18247288-37-32. [PubMed: 21745376].

20. Manzoni P. Hematologic Aspects of Early and Late-Onset Sepsis in Preterm Infants. Clin Perinatol. 2015;42(3):587-95. doi: 10.1016/j.clp.2015.04.012. [PubMed: 26250919].

21. Sheu JR, Hung WC, Wu CH, Ma MC, Kan YC, Lin CH, et al. Reduction in lipopolysaccharide-induced thrombocytopenia by triflavin in a rat model of septicemia. Circulation. 1999;99(23):3056-62. [PubMed: 10368125].

22. Sola MC, Calhoun DA, Hutson AD, Christensen RD. Plasma thrombopoietin concentrations in thrombocytopenic and nonthrombocytopenic patients in a neonatal intensive care unit. $\mathrm{Br} J$ Haematol.1999;104(1):90-2. [PubMed: 10027717].

23. Chen HN, Lee ML, Yu WK, Lin YW, Tsao LY. Late-onset Enterobacter cloacae sepsis in very-low-birth-weight neonates: experience in a medical center. Pediatr Neonatol. 2009;50(1):3-7. doi: 10.1016/S18759572(09)60022-X. [PubMed: 19326831].

24. Khashu M, Osiovich H, Henry D, Al Khotani A, Solimano A, Speert DP. Persistent bacteremia and severe thrombocytopenia caused by coagulase-negative Staphylococcus in a neonatal intensive care unit. Pediatrics. 2006;117(2):340-8. doi: 10.1542/peds.2005-0333. [PubMed: $16452352]$ 